https://doi.org/10.22319/rmcp.v12i4.5714

Nota de investigación

\title{
Digestibilidad ileal estandarizada de la proteína y aminoácidos de la pasta de ajonjolí en cerdos en crecimiento
}

Tércia Cesária Reis de Souza ${ }^{a}$

Araceli Aguilera Barreyro ${ }^{a}$

Gerardo Mariscal Landín ${ }^{\mathrm{b} *}$

anniversidad Autónoma de Querétaro. Facultad de Ciencias Naturales, Querétaro, México.

b Instituto Nacional de Investigaciones Forestales, Agrícolas y Pecuarias, CENID-Fisiología, Ajuchitlán 76280, Querétaro, México.

*Autor de correspondencia: mariscal.gerardo@inifap.gob.mx

\section{Resumen:}

Para determinar la digestibilidad ileal aparente (DIA) y estandarizada (DIE) de los aminoácidos de pasta de ajonjolí (PA), se utilizaron 10 cerdos de $78.6 \pm 5.2 \mathrm{~kg}$, alojados en jaulas metabólicas; localizadas en una sala con temperatura controlada (19 a $\left.22{ }^{\circ} \mathrm{C}\right)$. A los cerdos se les implantó una cánula " $T$ ” en íleon y se alimentaron dos veces al día, a 2.5 veces su requerimiento de energía digestible de mantenimiento $\left(110 \mathrm{kcal}\right.$ por $\mathrm{kg}$ de $\left.\mathrm{PV}^{0.75}\right)$. Se elaboraron dos dietas con $160 \mathrm{~g}$ de PC/kg de alimento: una con PA y otra con pasta de soya (PS). Los resultados muestran que la DIA de los siguientes aminoácidos fue superior en la PA que en la PS: arginina $(P<0.0001) 7.3$ unidades; alanina, ácido glutámico, glicina, metionina y valina fue en promedio 6.8 unidades mayor $(P<0.01)$; cisteína fue mayor en 11.5 unidades $(P<0.05)$. Contrariamente la DIA de prolina $(P<0.0001)$, leucina $(P<0.01)$ y lisina $(P<0.05)$ fueron inferiores en $21.9,2.8$ y 2.5 unidades respectivamente en la PA que en la PS. La DIE de arginina $(P<0.0001)$ fue $6.7 \%$ superior; valina $(P<0.001) 10.6 \%$ mayor, alanina, ácido glutámico, glicina y treonina $(P<0.01)$ fue superior en promedio en $6.4 \%$; y de cisteína, histidina, isoleucina, y tirosina $(P<0.05)$ fue superior en $7.45 \%$ en la PA que en la PS. La DIE de prolina $(P<0.01)$ y leucina $(P<0.05)$ fueron inferiores en 4.7 y 2.1 en la PA 
que en PS. Se concluye que la PA es una buena fuente de aminoácidos digestibles para la alimentación del cerdo.

Palabras clave: Pasta de Ajonjolí, Digestibilidad ileal, Aminoácidos, Cerdos.

Recibido: $24 / 06 / 2020$

Aceptado: 25/11/2020

El alto costo de la pasta de soya, ha propiciado la búsqueda de fuentes alternativas de proteína para ser utilizadas en la alimentación porcina ${ }^{(1)}$. Dentro de estas fuentes alternas, el ajonjolí se caracteriza por su alto contenido de aceite 40 a $50 \%$ y de proteína $27 \%$. Como subproducto de la extracción del aceite de ajonjolí se obtiene la pasta de ajonjolí, la cual se caracteriza por contener entre 45 y $50 \%$ de proteína, de 10 a $12 \%$ de extracto etéreo en la pasta obtenida por presión y entre 1 y $2 \%$ en la obtenida con solventes ${ }^{(3)}$. Esta pasta se caracteriza por ser una buena fuente de aminoácidos principalmente de aminoácidos azufrados, arginina y leucina; sin embargo, es pobre en lisina ${ }^{(3)}$. El uso de una fuente proteica depende de su aporte de aminoácidos, ya que los alimentos se formulan empleando los conceptos de proteína ideal $^{(4)}$ y de digestibilidad ileal estandarizada de los aminoácidos ${ }^{(5)}$, forma en que son expresados los requerimientos nutricionales del cerdo ${ }^{(6,7)}$. El uso de esos conceptos, y la disponibilidad de aminoácidos cristalinos (Lisina-HCL, L-Treonina, LTriptófano y DL-Metionina), han permitido la inclusión de diferentes fuentes proteicas en la alimentación del cerdo, aunque esas fuentes sean deficientes en alguno de esos aminoácidos, como sería el caso de la pasta de ajonjolí con respecto a la lisina. Sin embargo, la información disponible sobre la digestibilidad de la proteína y aminoácidos de la pasta de ajonjolí en cerdos es escasa y contradictoria, ya que se ha reportado una baja digestibilidad de los aminoácidos de ajonjolí en cerdos en crecimiento ${ }^{(8)}$. Por lo que el objetivo de este trabajo fue determinar la digestibilidad ileal en cerdos en crecimiento de los aminoácidos de pasta de ajonjolí obtenida por presión, ya que la información existente ha sido obtenida en pastas extraídas con solventes.

El trabajo se realizó en la granja experimental del CENID Fisiología del INIFAP. El protocolo fue revisado y aprobado por el Comité de Bioética de la Facultad de Ciencias Naturales de la Universidad Autónoma de Querétaro. El manejo empleado en los animales respetó los lineamientos de la Norma Oficial Mexicana para la producción, cuidado y uso de los animales de laboratorio ${ }^{(9)}$, así como los de la International Guiding Principles for Biomedical Research Involving Animals ${ }^{(10)}$. 
Se utilizaron 10 cerdos (machos castrados) provenientes de un cruzamiento (Landrace $\times$ Large-White), con un peso promedio de 78.6 $\pm 5.2 \mathrm{~kg}$ divididos en dos grupos de 5 cerdos. Los cerdos se alojaron en jaulas metabólicas individuales, provistas de comedero y bebedero; localizadas en un corral con temperatura controlada, la cual fluctuaba entre los 19 y $22{ }^{\circ} \mathrm{C}$. Los cuatro días posteriores a su ingreso a la unidad experimental sirvieron como periodo de adaptación a las jaulas metabólicas; durante los primeros tres días se les ofreció la dieta que consumían previamente; el cuarto día se ayunaron y en el quinto día se les implantó a los cerdos una cánula en "T" a nivel del íleon terminal ${ }^{(11)}$. El periodo postquirúrgico duró 21 días, en el cual los cerdos tuvieron libre acceso al agua y el alimento ofrecido durante ese periodo se incrementó gradualmente hasta alcanzar el consumo previo a la cirugía. Durante el periodo experimental los cerdos se alimentaron dos veces al día a razón de 2.5 veces su requerimiento de energía digestible de mantenimiento, el cual se estimó en 110 kcal por kilo de $\mathrm{PV}^{0.75(12)}$.

Se comparó la pasta de ajonjolí contra la pasta de soya (Cuadro 1). Con estas materias primas se formularon dos dietas experimentales (Cuadro 2), una dieta con pasta de ajonjolí (PA) y otra con pasta de soya (PS), ambas dietas aportaron $160 \mathrm{~g}$ de PC/kg de alimento; el nivel de inclusión de ambas pastas dependió de su contenido de proteína. A ambas dietas, se le agregó sacarosa a razón de $65 \mathrm{~g} / \mathrm{kg}$ para incrementar su palatibilidad, una fuente de fibra (Arbocel тм) $40 \mathrm{~g} / \mathrm{kg}$ y aceite de maíz a razón de $30 \mathrm{~g} / \mathrm{kg}$. Las vitaminas y minerales se adicionaron para proporcionar o exceder los requerimientos recomendados por el $\mathrm{NRC}^{(6)}$, el óxido de cromo se incluyó a razón de $3 \mathrm{~g} / \mathrm{kg}$ como marcador de digestibilidad. 
Cuadro 1: Composición de las materias primas

\begin{tabular}{lll}
\hline & Pasta de ajonjolí & Pasta de soya \\
\hline & & \\
Proteína cruda & 53.50 & 50.10 \\
Extracto etéreo & 11.10 & 1.30 \\
FDN & 17.50 & 13.80 \\
AIT, mg AIT/g ${ }^{1}$ & 1.00 & 6.00 \\
AF, g fitato de sodio/100g ${ }^{2}$ & 4.00 & 2.80 \\
& & \\
Alanina & 1.90 & 2.00 \\
Arginina & 4.90 & 3.40 \\
Ácido aspártico & 3.60 & 5.40 \\
Ácido glutámico & 7.50 & 9.30 \\
Glicina & 2.10 & 2.20 \\
Histidina & 1.00 & 1.20 \\
Isoleucina & 1.50 & 2.20 \\
Leucina & 2.80 & 3.40 \\
Lisina & 1.00 & 2.80 \\
Metionina + Cisteína & 2.10 & 1.40 \\
Fenilalanina & 1.80 & 2.50 \\
Prolina & 1.60 & 3.70 \\
Serina & 1.90 & 2.30 \\
Treonina & 1.50 & 1.90 \\
Tirosina & 1.60 & 1.80 \\
Valina & 1.90 & 2.30 \\
\hline & & \\
& &
\end{tabular}

${ }^{1} \mathrm{AIT}=$ actividad del inhibidor de tripsina.

${ }^{2} \mathrm{AF}=$ ácido fítico. 
Cuadro 2: Composición de las dietas experimentales

\begin{tabular}{|c|c|c|}
\hline & Pasta de ajonjolí & Pasta de soya \\
\hline Almidón de maíz & 53.60 & 46.37 \\
\hline Pasta de soya & & 36.40 \\
\hline Pasta de ajonjolí & 30.00 & \\
\hline Azúcar & 6.50 & 6.50 \\
\hline Celulosa $^{1}$ & 4.00 & 4.00 \\
\hline Aceite de maíz & 3.00 & 3.00 \\
\hline Sal & 0.40 & 0.40 \\
\hline Carbonato de calcio & 0.07 & 1.10 \\
\hline Fosfato dicálcico & 1.90 & 1.70 \\
\hline Premezcla mineral $^{2}$ & 0.07 & 0.07 \\
\hline Premezcla vitamínica ${ }^{3}$ & 0.16 & 0.16 \\
\hline Óxido de cromo & 0.30 & 0.30 \\
\hline \multicolumn{3}{|l|}{ Análisis químico: } \\
\hline Proteína cruda & 16.30 & 16.10 \\
\hline FDN & 8.80 & 11.90 \\
\hline
\end{tabular}

${ }^{1}$ Arbocel $=$ concentrado de fibra.

${ }^{2}$ Premezcla mineral $=$ aporta las siguientes cantidades por kilo de alimento: $\mathrm{Co}, 0.60 \mathrm{mg} ; \mathrm{Cu}, 14 \mathrm{mg} ; \mathrm{Fe}, 100$ $\mathrm{mg}$; I, $0.80 \mathrm{mg}$; Mn, $40 \mathrm{mg}$; Se, $0.25 \mathrm{mg}$; Zn, $120 \mathrm{mg}$.

${ }^{3}$ Premezcla vitamínica $=$ aporta las siguientes cantidades por kilo de alimento: vitamina A, 4,250 UI/g; vitamina D3, $800 \mathrm{UI} / \mathrm{g}$; vitamina E, $32 \mathrm{UI} / \mathrm{g}$; vitamina $\mathrm{K}_{3}$ menadiona, $1.5 \mathrm{mg} / \mathrm{kg}$; biotina, $120 \mathrm{mg} / \mathrm{kg}$; cianocobalamina, $16 \mu \mathrm{g} / \mathrm{kg}$; colina, $250 \mathrm{mg} / \mathrm{kg}$; ácido fólico, $800 \mathrm{mg} / \mathrm{kg}$; niacina, $15 \mathrm{mg} / \mathrm{kg}$; ácido pantoténico $13 \mathrm{mg} / \mathrm{kg}$; piridoxina $2.5 \mathrm{mg} / \mathrm{kg}$; riboflavina $5 \mathrm{mg} / \mathrm{kg}$; tiamina, $1.25 \mathrm{mg} / \mathrm{kg}$.

El agua se proporcionó a libertad a través de un bebedero de chupón localizado en una pared de la jaula metabólica. El periodo experimental tuvo una duración de siete días (cinco de adaptación a la dieta y dos para la colecta de la digesta ileal). La digesta ileal se colectó en bolsas de plástico (de $11 \mathrm{~cm}$ de largo $\times 5 \mathrm{~cm}$ de ancho), a las bolsas se les agregaban $10 \mathrm{ml}$ de una solución de $\mathrm{HCl} 0.2 \mathrm{M}$ con el objeto de bloquear toda actividad bacteriana. Las bolsas se fijaron a la cánula con una liga a las $0800 \mathrm{~h}$ del día uno y se colectó la digesta ileal de 0800 a $1800 \mathrm{~h}$. Conforme se llenaban las bolsas con la digesta ileal, ésta se transfería a un contenedor para proceder inmediatamente a congelarla a $-20{ }^{\circ} \mathrm{C}$ hasta su liofilización.

Las muestras de digesta del experimento se liofilizaron y posteriormente se molieron a través de una malla de $0.5 \mathrm{~mm}$ con un molino de laboratorio (Arthur H. Thomas Co. Philadelphia, PA). Los siguientes análisis se realizaron en las dietas experimentales y en las muestras de digesta ileal: materia seca (MS) y proteína cruda (PC) de acuerdo a los métodos 934.01 y 976.05 del $\mathrm{AOAC}^{(13)}$, óxido de cromo según Fenton y Fenton ${ }^{(14)}$. La preparación de las muestras para la determinación de AA se realizó siguiendo el método 994.12 del AOAC ${ }^{(13)}$, 
el cual consiste en hidrolizar las muestras a $110{ }^{\circ} \mathrm{C}$ durante $24 \mathrm{~h}$ en $\mathrm{HCl} 6 \mathrm{M}$; en el caso de metionina y cisteína se realizó una oxidación previa con ácido perfórmico. Los análisis de AA se realizaron por medio de cromatografía en fase reversa según el método descrito por Henderson et $a l^{(15)}$ en un HPLC de marca Hewlett Packard, modelo 1100. La actividad del inhibidor de tripsina (AIT) de las materias primas y las dietas experimentales se determinó de acuerdo con el método descrito por Kakade $e t a l^{(16)}$; el ácido fítico se analizó de acuerdo con Vaintraub y Lapteva ${ }^{(17)}$; y la fibra detergente neutra (FDN) se analizó según van Soest et $a l^{(18)}$.

Los cálculos para estimar la digestibilidad ileal aparente (DIA) de la proteína, aminoácidos y energía de las dietas experimentales, se realizaron empleando la ecuación utilizada por Fan y Sauer ${ }^{(19)}$.

$\mathrm{DIA}=[1-[(\mathrm{ID} \times \mathrm{AF}) /(\mathrm{AD} \times \mathrm{IF})]] \times 100$

Donde DAI es la digestibilidad aparente ileal de un nutrimento en la dieta en porcentaje, ID es la concentración del indicador en la dieta (mg/kg de MS), AF es la concentración del nutrimento en la digesta ileal ( $\mathrm{mg} / \mathrm{kg}$ de $\mathrm{MS}), \mathrm{AD}$ es la concentración del nutrimento en la dieta (mg/kg de MS), IF es la concentración del indicador en la digesta ileal (mg/kg de MS). Los cálculos para estimar la digestibilidad estandarizada ileal (DIE) de la proteína y aminoácidos se realizaron utilizando la fórmula propuesta por Furuya y Kaji ${ }^{(20)}$.

$\mathrm{DIE}=\mathrm{DIA}+[($ EndoN/ConsN $) \times 100]$

Donde DIE es la digestibilidad ileal estandarizada de un nutrimento en porcentaje. DIA es la digestibilidad ileal aparente de un nutrimento. EndoN es la cantidad endógena excretada del nutrimento en $\mathrm{mg} / \mathrm{kg}$ de materia seca consumida. ConsN es la cantidad de nutrimento consumido en $\mathrm{mg} / \mathrm{kg}$ de materia seca consumida.

Para los cálculos se utilizó el endógeno reportado por Mariscal-Landín y Reis de Souza ${ }^{(21)}$. Los datos de la DIA y DIE de la proteína y aminoácidos en cerdos en crecimiento se analizaron empleando el procedimiento GLM del paquete estadísticos $\mathrm{SAS}^{(22)}$, según un diseño completamente al azar ${ }^{(23)}$. Las medias de tratamientos se compararon empleando el método de Tukey ${ }^{(23)}$. Las diferencias fueron consideradas significativas cuando $(P<0.05)$, y una tendencia fue reconocida cuando $(0.05<P<0.10)$.

Los resultados muestran que los cerdos consumieron su ración completa. La pasta de ajonjolí tuvo un $6.8 \%$ más PC; $42.9 \%$ más ácido fítico y $26.8 \%$ más FDN que la pasta de soya. El contenido de extracto etéreo de la pasta de ajonjolí fue 8.5 veces superior $(111 \mathrm{vs} 13 \mathrm{~g} / \mathrm{kg})$ al de la pasta de soya (Cuadro 1). Por el contrario, el contenido del inhibidor de tripsina fue 6 veces superior en la pasta de soya $(6 \mathrm{mg} / \mathrm{g})$ que en la pasta de ajonjolí $(1 \mathrm{mg} / \mathrm{g})$. En lo que 
respecta al contenido de aminoácidos totales la pasta de ajonjolí tuvo un 50 \% más contenido de aminoácidos azufrados (metionina + cisteína) y un $44 \%$ más de arginina que la pasta de soya. Por el contrario, la pasta de soya tuvo un contenido de lisina $280 \%$ superior que la pasta de ajonjolí, al igual que en el caso de la prolina $231 \%$; así como un $50 \%$ más de ácido aspártico, $46 \%$ más de isoleucina, $38 \%$ más de fenilalanina y $26 \%$ más de treonina.

La DIA de la proteína cruda de la pasta de ajonjolí fue mayor $(P<0.01)$ en 5.6 unidades porcentuales que la de pasta de soya (Cuadro 3). La DIA de la arginina fue superior $(P<0.0001)$ en 7.3 unidades porcentuales en la pasta de ajonjolí que en la pasta de soya; las digestibilidades de alanina, ácido glutámico, glicina, metionina y valina fueron en promedio 6.8 unidades porcentuales mayores $(P<0.01)$ en la pasta de ajonjolí que en la pasta de soya. La DIA de cisteína, fue mayor en 11.5 unidades porcentuales $(P<0.05)$ en la pasta de ajonjolí que en pasta de soya. Por el contrario, la DIA de prolina $(P<0.0001)$, así como la de leucina $(P<0.01)$ y de lisina $(P<0.05)$ fueron inferiores en $21.9,2.8$ y 2.5 unidades porcentuales respectivamente en la pasta de ajonjolí que en la pasta de soya (Cuadro 3).

Cuadro 3: Digestibilidad ileal aparente

\begin{tabular}{|c|c|c|c|c|}
\hline & $\begin{array}{l}\text { Pasta de } \\
\text { ajonjolí }\end{array}$ & Pasta de soya & Probabilidad & EEM \\
\hline Proteína cruda & $83.8^{a}$ & $78.2 \mathrm{~b}$ & 0.01 & 0.8 \\
\hline Alanina & $76.1^{\mathrm{a}}$ & $67.8^{b}$ & 0.01 & 0.8 \\
\hline Arginina & $92.9^{\mathrm{a}}$ & $85.6^{\mathrm{b}}$ & 0.0001 & 0.4 \\
\hline Ácido aspártico & 83.6 & 85.3 & NS & 0.4 \\
\hline Cisteína & $72.9^{\mathrm{a}}$ & $61.6^{b}$ & 0.05 & 2.3 \\
\hline Ácido glutámico & $90.5^{\mathrm{a}}$ & $87.1^{\mathrm{b}}$ & 0.01 & 0.4 \\
\hline Glicina & $82.1^{\mathrm{a}}$ & $77.0^{b}$ & 0.01 & 0.6 \\
\hline Histidina & $90.8^{\mathrm{a}}$ & $87.1^{\mathrm{b}}$ & 0.05 & 0.7 \\
\hline Isoleucina & 82.8 & 81.4 & NS & 0.5 \\
\hline Leucina & $84.4^{b}$ & $87.2^{\mathrm{a}}$ & 0.01 & 0.4 \\
\hline Lisina & $91.6^{b}$ & $94.1^{\mathrm{a}}$ & 0.05 & 0.4 \\
\hline Metionina & $83.4^{\mathrm{a}}$ & $72.4^{b}$ & 0.01 & 1.3 \\
\hline Fenilalanina & 82.2 & 81.2 & NS & 0.5 \\
\hline Prolina & $59.6^{\mathrm{b}}$ & $81.5^{\mathrm{a}}$ & 0.0001 & 0.5 \\
\hline Serina & 84.1 & 83.6 & NS & 1 \\
\hline Treonina & 76.9 & 76.0 & NS & 0.4 \\
\hline Tirosina & 76.2 & 72.9 & NS & 0.7 \\
\hline Valina & $78.5^{\mathrm{a}}$ & $72.0^{\mathrm{b}}$ & 0.01 & 0.7 \\
\hline
\end{tabular}


La DIE de la proteína de la pasta de ajonjolí fue mayor $(P<0.01)$ en un $6.4 \%$. La DIE de la arginina $(P<0.0001)$ fue $6.7 \%$ superior; de la valina $(P<0.001) 10.6 \%$ mayor, de los aminoácidos alanina, ácido glutámico, glicina y treonina $(P<0.01)$ fue superior en promedio en $6.4 \%$; y la DIE de cisteína, histidina, isoleucina, y tirosina $(P<0.05)$ fue superior en un $7.45 \%$ en la pasta de ajonjolí que en la pasta de soya. Sin embargo, la DIE de la prolina $(P<0.01)$ y la leucina $(P<0.05)$ fueron inferiores en 4.7 y $2.1 \%$ en la pasta de ajonjolí con respecto a las de la pasta de soya (Cuadro 4).

Cuadro 4: Digestibilidad ileal estandarizada

\begin{tabular}{lllll}
\hline & $\begin{array}{c}\text { Pasta de } \\
\text { ajonjolí }\end{array}$ & $\begin{array}{c}\text { Pasta de } \\
\text { soya }\end{array}$ & Probabilidad & EEM \\
\hline Proteína cruda & $91.6^{\mathrm{a}}$ & $86.1^{\mathrm{b}}$ & 0.01 & 0.8 \\
& & & & \\
Alanina & $83.4^{\mathrm{a}}$ & $75.2^{\mathrm{b}}$ & 0.01 & 0.8 \\
Arginina & $95.8^{\mathrm{a}}$ & $89.8^{\mathrm{b}}$ & 0.0001 & 0.4 \\
Ácido aspártico & 88.3 & $88.4^{\mathrm{a}}$ & $\mathrm{NS}$ & 0.4 \\
Cisteína & $75.9^{\mathrm{a}}$ & $65.0^{\mathrm{b}}$ & 0.05 & 2.3 \\
Ácido glutámico & $94.2^{\mathrm{a}}$ & $90.5^{\mathrm{b}}$ & 0.01 & 0.4 \\
Glicina & $88.8^{\mathrm{a}}$ & $83.5^{\mathrm{b}}$ & 0.01 & 0.6 \\
Histidina & $93.8^{\mathrm{a}}$ & $90.2^{\mathrm{b}}$ & 0.05 & 0.7 \\
Isoleucina & $90.5^{\mathrm{a}}$ & $87.3^{\mathrm{b}}$ & 0.05 & 0.5 \\
Leucina & $89.4^{\mathrm{b}}$ & $91.3^{\mathrm{a}}$ & 0.05 & 0.4 \\
Lisina & 95.5 & $96.2^{2}$ & $\mathrm{NS}$ & 0.4 \\
Metionina & 86.3 & 76.2 & $\mathrm{NS}$ & 1.3 \\
Fenilalanina & 86.00 & $84.2^{\mathrm{a}}$ & $\mathrm{NS}$ & 0.5 \\
Prolina & $86.3^{\mathrm{b}}$ & $90.6^{\mathrm{a}}$ & 0.01 & 0.5 \\
Serina & 94.0 & 91.5 & $\mathrm{NS}$ & 1 \\
Treonina & $88.2^{\mathrm{a}}$ & $84.5^{\mathrm{b}}$ & 0.01 & 0.4 \\
Tirosina & $81.6^{\mathrm{a}}$ & $77.6^{\mathrm{b}}$ & 0.05 & 0.7 \\
Valina & $86.7^{\mathrm{a}}$ & $78.4^{\mathrm{b}}$ & 0.001 & 0.7 \\
\hline
\end{tabular}

EEM= Error estándar de la media.

Según la FAO la producción mundial de ajonjolí en 2018 fue de 6’448,961 ton métricas, ocupando México el quinceavo lugar con una producción de 57,256 t, FAO STAT ${ }^{(24)}$. El ajonjolí se cultiva principalmente como fuente de aceite, ya que contiene en promedio 44 a $58 \%$ de aceite, pero también es rico en proteína 18 a $25 \% \mathrm{PC}^{(25)}$. El aceite de ajonjolí se caracteriza por ser muy estable, debido a la presencia de los antioxidantes naturales (sesamolina, sesamina y sesamol) ${ }^{(26)}$. Las proteínas del ajonjolí consisten predominantemente de cuatro fracciones proteicas, las cuales son designadas en función de su peso molecular como $2 \mathrm{~S}, 7 \mathrm{~S}, 11 \mathrm{~S}$ (bajo, medio y alto peso molecular) y 15-18S (proteínas poliméricas resultantes de una posible agregación de $2 \mathrm{~S}, 7 \mathrm{~S}$ u $11 \mathrm{~S})^{(27)}$. Las proteínas de alto 
peso molecular son las principales en el ajonjolí, y ellas se caracterizan por ser ricas en ácido glutámico y aminoácidos aromáticos, y bajas en lisina; además de tener una baja proporción de la conformación $\alpha$-hélice y una alta proporción de lámina $\beta$ eta $^{(27)}$; la proteína del ajonjolí se caracteriza por ser rica en arginina ${ }^{(28)}$. La globulina $11 \mathrm{~S}$ (insoluble en agua) y la albúmina $2 \mathrm{~S}$ (soluble), se denominan $\alpha$-globulina y $\beta$-globulina respectivamente; y son las dos principales proteínas de almacenamiento del ajonjolí, constituyendo entre el 80 y el $90 \%$ del total de las proteínas de ajonjolí ${ }^{(29)}$.

Se ha reportado que el ácido fítico puede interferir con la digestibilidad de la proteína debido a su capacidad quelante ${ }^{(30)}$. El fitato se forma durante el período de maduración de la planta y su función es ser almacenamiento de $\mathrm{P}$ y minerales, jugando un papel importante en el metabolismo de las semillas durante la germinación. Además, el mioinositol (el componente químico de la molécula de fitato) es utilizado para la formación de las paredes celulares ${ }^{(31)}$. El ajonjolí se caracteriza por contener niveles altos de fitato, (14.6 g de fitato-P/kg de semilla, hasta $51.8 \mathrm{~g}$ de fitato/kg de pasta ${ }^{(28)}$. La molécula de fitato contiene doce protones reactivos, seis pueden disociarse a $\mathrm{pH}$ ácido, tres a $\mathrm{pH}$ neutro y los tres restantes a pH básico, lo que le permite unirse con moléculas cargadas provenientes de la dieta y con secreciones endógenas como las enzimas digestivas y la mucina en todas las condiciones de $\mathrm{pH}$ encontrado en el intestino $^{(32)}$. Entre los aminoácidos que son quelados más fácilmente por el fitato están los aminoácidos básicos y ya se mencionó previamente la riqueza en arginina de la proteína del ajonjolí $^{(28,30)}$. Sin embargo, la digestibilidad en pepsina del aislado de proteína de ajonjolí es alta $89.57 \%$; por lo que se puede asumir que lo que modularía negativamente la digestibilidad de la proteína de ajonjolí es la cantidad de fítato.

La DIA de la proteína fue de 83.8 y la DIA promedio de los aminoácidos del ajonjolí fue de 81.7, muy similar a la DIA promedio de los aminoácidos de pasta de soya, la cual fue de 79.6; sin embargo, se observaron diferencias en algunos aminoácidos siendo superiores la DIA de leucina, lisina y prolina en el caso de pasta de soya. En el caso particular de lisina su mayor DIA puede ser debida al mayor contenido de lisina en la pasta de soya: 2.8 veces superior con respecto a la de la pasta de ajonjolí. Similar situación se observa en el caso de arginina (ya que la DIA de arginina en la pasta de ajonjolí fue superior en 7.3 unidades porcentuales), metionina (la DIA en la pasta de ajonjolí fue superior en 11.0 unidades porcentuales) y cisteína (la DIA en la pasta de ajonjolí fue superior en 11.3 unidades porcentuales). En los tres casos el ajonjolí fue más rico: arginina $30.6 \%$; aminoácidos azufrados $50.0 \%$. La DIA de la proteína y aminoácidos reportados en el presente trabajo son similares a los reportados previamente ${ }^{(33,34,35)}$.

En lo que respecta a la DIE de la proteína y aminoácidos del ajonjolí, esta fue mayor en 5.5 y 3.8 unidades porcentuales respectivamente, manteniéndose la diferencia en la DIE de arginina, la cual fue mayor en 6 unidades porcentuales en ajonjolí que en pasta de soya y en el caso de la DIE de lisina, esta fue similar en el ajonjolí y la pasta de soya; de igual manera 
la DIE de los aminoácidos de ajonjolí reportados en el presente trabajo son similares a los reportados previamente ${ }^{(33,34,35)}$. La diferencia encontrada en la DIE de la proteína y aminoácidos de la pasta de ajonjolí reportada por Son et $a l^{(8)}$ pudiera ser debida a la calidad de la pasta de ajonjolí que ellos utilizaron en su estudio, ya que los mismos autores mencionan que la baja digestibilidad pudo ser debido a un diferente proceso de extracción del aceite (sin particularizar en el mismo); y al contenido de FDN, ya que fue mucho más rica en FDN la pasta de ajonjolí utilizada por Son $e a l^{(8)}$, la cual contenía $28 \%$ de FDN y la utilizada en este trabajo contenía 10 unidades porcentuales menos de FDN (17.5\%); aunque se sabe que la fibra incrementa las pérdidas endógenas de aminoácidos ${ }^{(36,37)}$, afectando en algunos casos la digestibilidad ileal de los mismos ${ }^{(36)}$. Sin embargo, a pesar de su alto contenido de fitatos, la buena digestibilidad de la proteína y aminoácidos de la pasta de ajonjolí permite utilizarla en la alimentación de $\operatorname{cerdos}^{(38)}$ y aves ${ }^{(39)}$ en cualquier etapa productiva, sin menoscabo del aspecto productivo.

Los resultados permiten concluir que la pasta de ajonjolí es una fuente alternativa de proteína y aminoácidos digestibles para la alimentación del cerdo, ya que la DIE promedio de sus aminoácidos es de $88.5 \%$. Además de ser una fuente rica de arginina y aminoácidos azufrados.

\section{Agradecimientos}

Este estudio fue financiado parcialmente por la Universidad Autónoma de Querétaro a través del Fondo de Fortalecimiento para la Investigación, y el Instituto Nacional de Investigaciones Forestales, Agrícolas y Pecuarias. Los autores agradecen a Dipasa Internacional de México, S.A. de C.V. por su ayuda en proporcionar la pasta de ajonjolí utilizada en este estudio.

\section{Literatura citada:}

1. Woyengo TA, Beltranena E, Zijlstra RT. Nonruminant Nutrition Symposium: Controlling feed cost by including alternative ingredients into pig diets: A review. J Anim Sci 2014;92:1293-1305.

2. Nzikou JM, Matos L, Bouanga-Kalou G, Ndangui CB, Pambou-Tobi NPG, Kimbonguila A, et al. Chemical composition on the seeds and oil of sesame (Sesamum indicum L.) grown in Congo-Brazzaville. Ad J Food Sci Technol 2009;1:6-11.

3. Aguilera A, Reis de Souza TC, Mariscal-Landín G, Escobar K, Montaño S, Bernal MG. Standardized ileal digestibility of proteins and amino acids in sesame expeller and soya bean meal in weaning piglets. J Anim Physiol Anim Nutr 2015;99:728-736. 
4. Wang TC, Fuller MF. The optimum dietary amino acid pattern for growing pigs 1. Experiments by amino acid deletion. Br J Nutr 1989;62:77-89.

5. Stein HH, Sève B, Fuller MF, Moughan PJ, de Lange CFM. Invited review: Amino acid bioavailability and digestibility in pig feed ingredients: Terminology and application. $\mathrm{J}$ Anim Sci 2007;85:172-180.

6. NRC. Nutrient Requirements of Swine: Eleventh Rev Ed. Washington, DC: The National Academies Press; 2012.

7. INRA. Tables de composition et de valeur nutritive des matières premières destinées aux animaux d'élevage. Porcs, volailles, bovins, ovins, caprins, lapins, chevaux, poissons. Paris, France: Institut National de la Recherche Agronomique; 2002.

8. Son AR, Park CS, Park KR, Kim BG. Amino acid digestibility in plant protein sources fed to growing pigs. Asian Australas J Anim Sci 2019;32:1745-1752.

9. Diario Oficial de la Federación. Especificaciones técnicas para la producción, cuidado y uso de los animales de laboratorio. Norma Oficial Mexicana NOM-062-ZOO-1999. Diario Oficial de la Federación 2001(Miércoles 2 de agosto).

10. CIOMS. International guiding principles for biomedical research involving animals. In: Organization WH editor. International guiding principles for biomedical research involving animals. Council for International Organizations of Medical Sciences ed. Geneva; 1985.

11. Reis de Souza TC, Mar BB, Mariscal LG. Canulación de cerdos posdestete para pruebas de digestibilidad ileal: Desarrollo de una metodología. Téc Pecu Méx 2000;38:143-150.

12. INRA. L'alimentation des animaux monogastriques: porc, lapin, volailles. Paris, France: Institut National de la Recherche Agronomique; 1984.

13. AOAC. Official Methods of Analysis. 17 th. ed. Arlington, VA. USA: Assoc Offic Anal Chem; 2000.

14. Fenton TW, Fenton M. An improved procedure for determination of chromic oxide in feed and feces. Can J Anim Sci 1979;59:631-634.

15. Henderson JH, Ricker RD, Bidlingmeyer BA, Woodward C. Rapid, accurate and reproducible HPLC analysis of amino acids. Amino acid analysis using Zorbax Eclipse AAA columns and the Agilent 1100 HPLC. Agilent technologies 2000(Part No.59801193E):10 pag. Agilent technologies home page at: www.agilent.com/chem/supplies. 
16. Kakade ML, Rackis JJ, McGhee JE, Puski G. Determination of trypsin inhibitor activity of soy products: A collaborative analysis of an improved procedure. Cereal Chem 1974;51:376-382.

17. Vaintraub IA, Lapteva NA. Colorimetric determination of phytate in unpurified extracts of seeds and the products of their processing. Anal Biochem 1988;15:227-230.

18. van Soest PJ, Robertson JB, Lewis BA. Methods for dietary fiber, neutral detergent fiber, and nonstarch polysaccharides in relation to animal nutrition. J Dairy Sci 1991;74:3583-3597.

19. Fan MZ, Sauer WC. Determination of apparent ileal amino acid digestibility in barley and canola meal for pigs with the direct, difference, and regression methods. J Anim Sci 1995;73:2364-2374.

20. Furuya S, Kaji Y. Estimation of the true ileal digestibility of amino acids and nitrogen from their apparent values for growing pigs. Anim Feed Sci Technol 1989;26:271-285.

21. Mariscal-Landín G, Reis de Souza TC. Endogenous ileal losses of nitrogen and amino acids in pigs and piglets fed graded levels of casein. Arch Anim Nutr 2006;60:454-466.

22. SAS version 9.2. Statistical Analysis Systems Institute User's guide. Statistical Analysis Systems Institute User's guide. 9.2 ed. Cary NC, USA: SAS Institute Inc.; 2008.

23. Steel RGD, Torrie JH. Principles and procedures of statistics. A Biometrical approach. 2nd ed. New York: McGraw-Hill 1980.

24. FAO. "Food Outlook, Bienal Report on Global Food Markets," Bienal Report on Global Food Markets. Date acces: 14-Sep-2020. http://www.fao.org/3/ca9509en/ca9509en.pdf.

25. Omer HAA, Ahmed SM, Abdel-Magid SS, Bakry A, El-Karamany MF, El-Sabaawy EH. Nutritional impact of partial or complete replacement of soybean meal by sesame (Sesamum indicum) meal in lambs rations. Bull Natl Res Cent 2019;43:98.

26. Dossa K, Diouf D, Wang L, Wei X, Zhang Y, Niang M, et al. The emerging oilseed crop sesamum indicum enters the "Omics” era. Front Plant Sci 2017;8:1154.

27. Prakash V, Rao M. Structural similarities among the high molecular weight protein fractions of oilseeds. J Biosci 1988;13:171-180.

28. Selle P, Cowieson A, Cowieson N, Ravindran V. Protein-phytate interactions in pig and poultry nutrition: a reappraisal. Nutr Res Rev 2012;25:1-17.

29. Orruño E, Morgan MRA. Purification and characterisation of the $7 \mathrm{~S}$ globulin storage protein from sesame (Sesamum indicum L.). Food Chem 2007;100:926-934. 
30. Selle PH, Ravindran V, Caldwell A, Bryden WL. Phytate and phytase: consequences for protein utilisation. Nutr Res Rev 2000;13:255-278.

31. Humer E, Schwarz C, Schedle K. Phytate in pig and poultry nutrition. J Anim Physiol Anim Nutr 2015;99:605-625.

32. Woyengo TA, Cowieson AJ, Adeola O, Nyachoti CM. Ileal digestibility and endogenous flow of minerals and amino acids: responses to dietary phytic acid in piglets. Br J Nutr 2009;102:428-433.

33. Fasuan TO, Gbadamosi SO, Omobuwajo TO. Characterization of protein isolate from Sesamum indicum seed: In vitro protein digestibility, amino acid profile, and some functional properties. Food Sci Nutr 2018;6:1715-1723.

34. Li D, Qiao SY, Yi GF, Jiang JY, Xu XX, Piao XS, et al. Performance of growingfinishing pigs fed sesame meal supplemented diets formulated using amino acid digestibilities determined by the regression technique. Asian Australas J Anim Sci 2000;13:213-219.

35. Casas GA, Jaworski NW, Htoo JK, Stein HH. Ileal digestibility of amino acids in selected feed ingredients fed to young growing pigs. J Anim Sci 2018;96:2361-2370.

36. Mariscal-Landín G, Reis de Souza TC, Hernández DAA, Escobar GK. Pérdidas endógenas de nitrógeno y aminoácidos en cerdos y su aplicación en la estimación de los coeficientes de digestibilidad ileal de la proteína y aminoácidos de las materias primas. Téc Pecu Méx 2009;47:371-388.

37. Mariscal-Landín G, Reis de Souza TC, Bayardo UA. Neutral detergent fiber increases endogenous ileal losses but has no effect on ileal digestibility of amino acids in growing pigs. Anim Sci J 2017;88:322-330.

38. Reis de Souza TC, Escobar García K, Aguilera AB, Ramirez RE, Mariscal-Landín G. Sesame meal as the first protein source in piglet starter diets and advantages of a phytase: a digestive study. S Afr J Anim Sci 2017;47:606-615.

39. Amin H, Majid M, Maryam H. Effects of microbial fermented sesame meal and enzyme supplementation on the intestinal morphology, microbiota, $\mathrm{pH}$, tibia bone and blood parameters of broiler chicks. Ital J Anim Sci 2020;19:457-467. 